\title{
Hypertrophic cardiomyopathy with paroxysmal atrial fibrillation misdiagnosed as WPW syndrome
}

\author{
Sang-Hoon Seol, Ki-Hun Kim ${ }^{*}$, Jino Park, Yeo-Jeong Song, Dong-Kie Kim and Doo-II Kim
}

\begin{abstract}
Hypertrophic cardiomyopathy (HCM) is associated with an increased incidence of Wolff-Parkinson-White (WPW) syndrome and atrial fibrillation. However, a delta-like wide QRS can be observed in the hypertrophied myocardium. When considering the rarity of the paraseptal bypass tract (BT), the normal QRS axis suggests a higher possibility of HCM origin. Otherwise, there is no known electrocardiographic clue indicating a wide QRS differentiation between HCM and WPW syndrome. Moreover, the atriofascicular, nodofascicular/ventricular or fasciculoventricular BT should be differentiated. In this case, atrioventricular conduction system incidental injury revealed a wide QRS origin from the HCM, but this method should be avoided except in some selected cases.
\end{abstract}

Keywords: Atrial fibrillation, Hypertrophic cardiomyopathy, WPW syndrome

\section{Introduction}

Hypertrophic cardiomyopathy (HCM) is associated with an increased incidence of Wolff-Parkinson-White (WPW) syndrome and atrial fibrillation (AF). However, a delta-like wide QRS can be observed in the hypertrophied myocardium. When considering the rarity of the paraseptal bypass tract (BT), the normal QRS axis suggests a higher possibility of HCM origin. Otherwise, there is no known electrocardiographic clue indicating a wide QRS differentiation between HCM and WPW syndrome. Moreover, the atriofascicular, nodofascicular/ventricular or fasciculoventricular BT should be differentiated.

\section{Case report}

A 54-year-old man was admitted to our emergency room with palpitation for $6 \mathrm{~h}$. He experienced the same symptoms 8 years ago and was diagnosed with HCM and WPW syndrome with paroxysmal AF. Atorvastatin,

*Correspondence: ironkh@yahoo.co.kr

Division of Cardiology, Department of Internal Medicine, Haeundae Paik Hospital, Inje University College of Medicine, 875 Haeundae-ro, Haeundae-gu, Busan 48108, Korea perindopril, rivaroxaban, sotalol and torsemide were prescribed. His blood pressure was $80 / 50 \mathrm{mmHg}$, heart rate was $169 \mathrm{bpm}$, and respiratory rate was 20 breaths/ minutes. His electrocardiogram showed irregular wide QRS tachycardia (normal QRS axis, left bundle branch block morphology and R/S transition at V3) (Fig. 1). After DC cardioversion, marked sinus bradycardia with a short PR interval (86 ms) and delta wave was observed. The QRS axis and morphology did not change (Fig. 2a). His echocardiography showed reduced left ventricular (LV) ejection fraction $(50 \%)$, marked thickening of the LV wall, and restrictive physiology of the LV filling pattern. An electrophysiological study was performed for recurrent symptoms and hemodynamic deterioration. Baseline atrial-His $(\mathrm{AH})$ interval was $53 \mathrm{~ms}$ and His-ventricular (HV) interval was $35 \mathrm{~ms}$ (Fig. 2b). Concentric ventriculoatrial activation pattern was observed during right ventricle (RV) pacing, and decremental conduction property could not be evaluated due to early atrioventricular (AV) conduction block (S1/S2 cycle length (CL) 450/320 ms). High right atrium (HRA) pacing showed persistent wide 


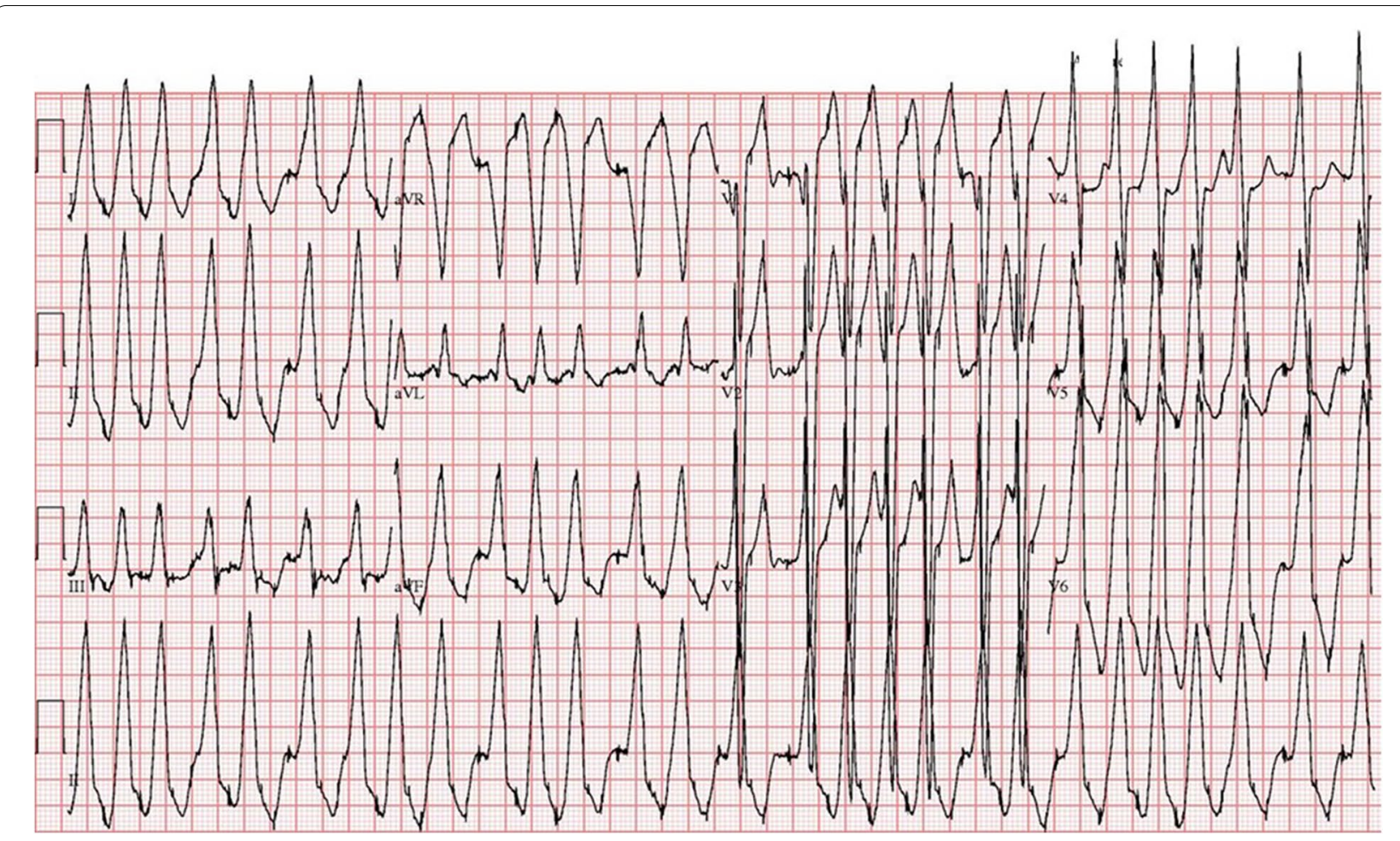

Fig. 1 Electrocardiogram showed irregular wide QRS tachycardia (normal QRS axis, left bundle branch block morphology and R/S transition at V3)

QRS morphology. Supraventricular or other arrhythmias were not induced during baseline, isoproterenol infusion (up to $3 \mathrm{mcg} / \mathrm{min}$ ) and washout period. Three-D activation mapping (EnSite Velocity ${ }^{\mathrm{TM}}$, Abbott, USA) showed the earliest ventricular activation site near the His bundle during HRA pacing (red dot; His bundle area, white spot; the earliest ventricular activation site) (Fig. 3a). Because AV conduction via the midseptal BT could not be excluded and permanent pacemaker implantation was planned for his sick sinus syndrome, catheter ablation targeting the earliest ventricular activation site was attempted. Several ablations $(10 \mathrm{~W})$ using a non-irrigated ablation catheter at the earliest ventricular activation site (white spot) had no effect. Meanwhile, sudden AV conduction delay (PR interval from 86 to $216 \mathrm{~ms}$ ) mainly due to prolongation of the $\mathrm{AH}$ interval (from 53 to $114 \mathrm{~ms}$ ) occurred by the ablation under the His bundle (light blue dot).
The QRS morphology was changed in lead III (from Rs to rS) and aVF (from Rs to RS), but the delta-like wide QRS was persisted (Fig. 3b-c). We stopped the catheter ablation and performed cryoablation for the AF. Four pulmonary veins were successfully isolated. However, the patient experienced syncope 3 months later, and paroxysmal AV block was diagnosed. Finally, a permanent pacemaker was implanted and followed up to date without symptoms.

\section{Discussion}

HCM is commonly inherited primary cardiac disorder and associated with an increased incidence of WPW syndrome and $10-40 \%$ of AF [1-3]. However, a deltalike wide QRS morphology could be observed in the hypertrophied myocardium due to the intraventricular conduction delay. To the best of our knowledge, there is no electrocardiogram interpretation method for 


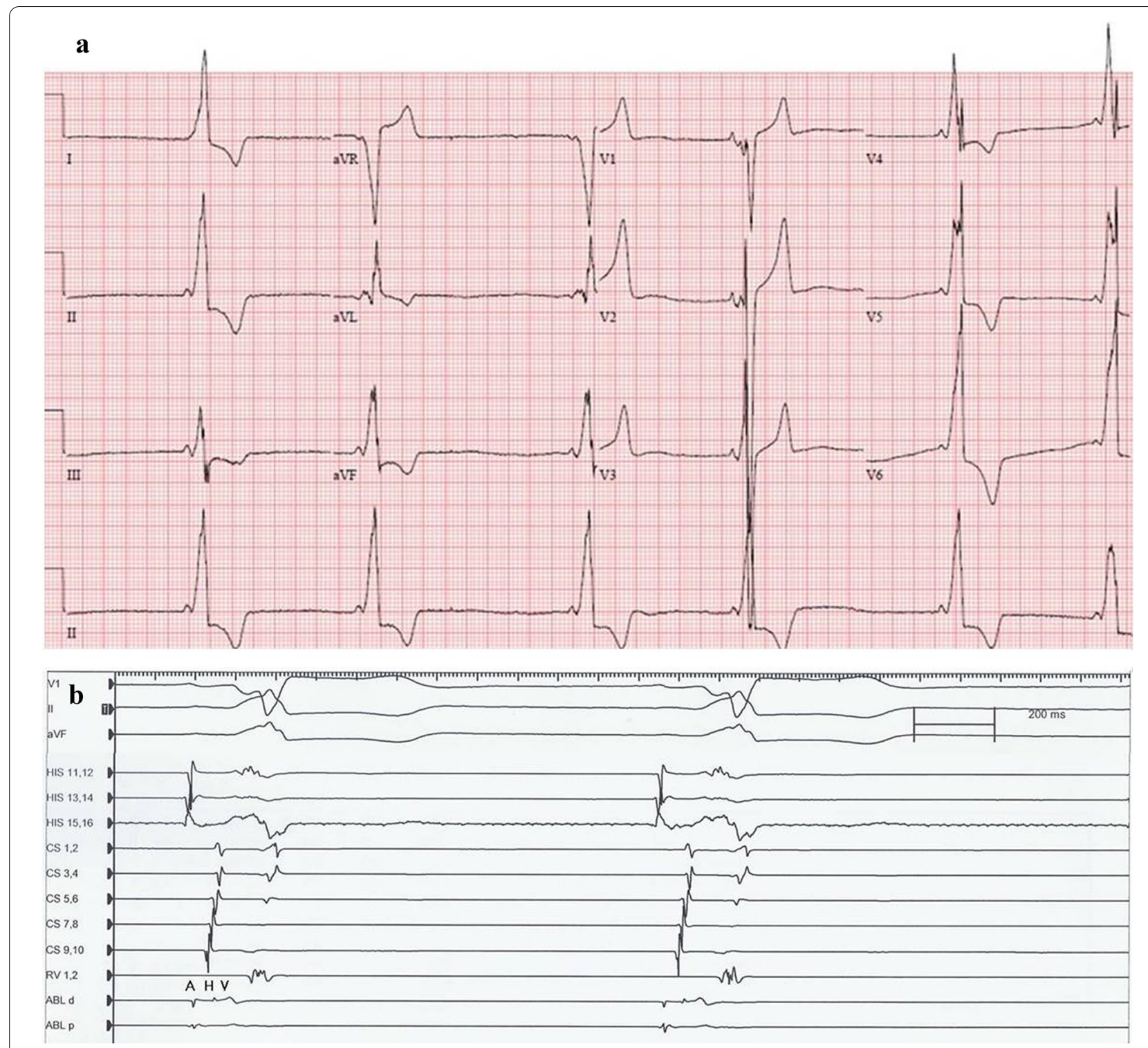

Fig. 2 a After DC cardioversion, marked sinus bradycardia with short PR interval (86 ms) and delta wave was observed. The QRS axis and morphology was not changed. b The ablation catheter was positioned on the His bundle (red dot in Fig. 3). Baseline AH interval was 53 ms and HV interval was 35 ms. A: atrium, ABL: ablation catheter (d: distal, p: proximal), CS: coronary sinus (9, 10: proximal site, 1, 2: distal site), H and HIS: His bundle, RV: right ventricle, V: ventricle

the differentiation of wide QRS due to HCM or WPW syndrome.

Although QRS morphologies and axis in some limb leads were slightly changed by the catheter ablation, the delta-like wide QRS was persisted in this case. The changing of QRS morphologies in the lead III (from Rs to
$\mathrm{rS}$ ) and aVF (from Rs to RS) might be related to changing of the intraventricular conduction rather than ablation of the BT, suggesting that the hypertrophied myocardium causes the delta-like QRS widening. When considering the rarity of paraseptal BT, the normal QRS axis could be a clue of HCM cause. In such a case, further detailed 
evaluation including electrophysiology study will be required, and selection of the catheter ablation should be prudent. Furthermore, differential diagnosis for septal BT should be performed before the catheter ablation. We tried to evaluate decremental property from the RV pacing, but decremental conduction was not observed due to early AV conduction block and HRA pacing showed persistent wide QRS morphology, which might suggest septal BT. However, PR interval was prolonged by the catheter ablation mainly due to prolongation of $\mathrm{AH}$ interval and partially $\mathrm{HV}$ interval combined some QRS morphologic changes. This finding is compatible with AV conduction delay rather than the ablation of septal BT for the prolongation of PR interval. Para-Hisian pacing could be helpful for differentiation between septal BT and delay conduction by HCM. Additionally, atriofascicular, nodofascicular/ventricular or fasciculoventricular BT should be differentiated [4].

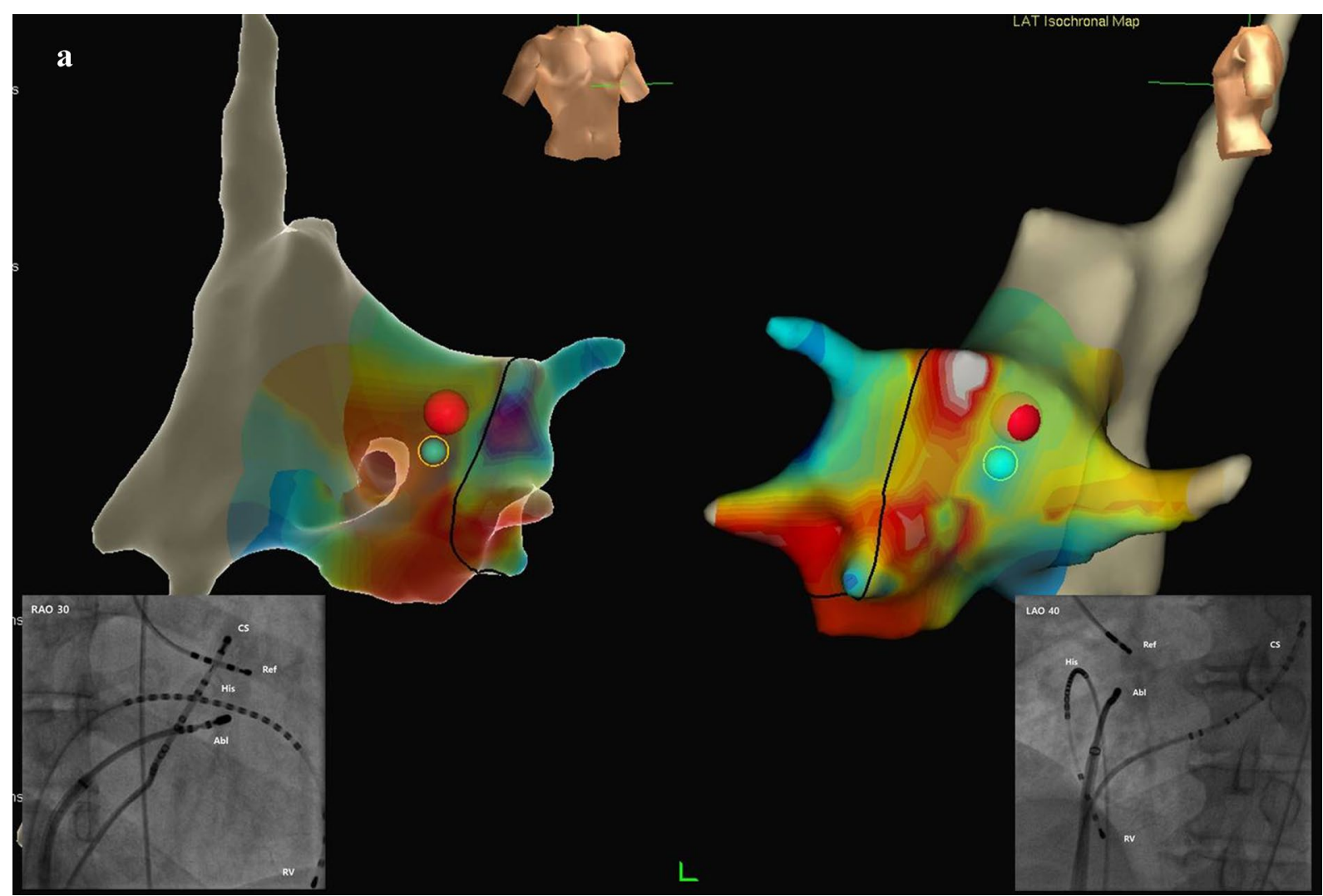

Fig. 3 a Three-D activation mapping showed the earliest ventricular activation site near the His bundle during HRA pacing (black line; suspected tricuspid valve area, light blue dot; ablation site making prolongation of AV conduction, red dot; His bundle area, white spot; the earliest ventricular activation site). The ablation catheter was positioned under the His bundle when sudden AV conduction delay occurred. Left 3D and angiographic image is taken by RAO 30 degree and right 3D and angiographic image is taken by LAO 40 degree. b PR interval was prolonged from 86 to $216 \mathrm{~ms}$ by the ablation under the His bundle. The QRS morphologies were changed in lead III (from Rs to rS) and aVF (from Rs to RS), but the delta-like wide QRS was persisted. c The AH interval was prolonged from 53 to $114 \mathrm{~ms}$ and the HV interval was from 35 to $61 \mathrm{~ms}$. ABL: ablation catheter (d: distal, p: proximal), CS: coronary sinus (9, 10: proximal site, 1, 2: distal site), H and HIS: His bundle, LAO: left anterior oblique, RAO: right anterior oblique, Ref: reference catheter, RV: right ventricle, V: ventricle 


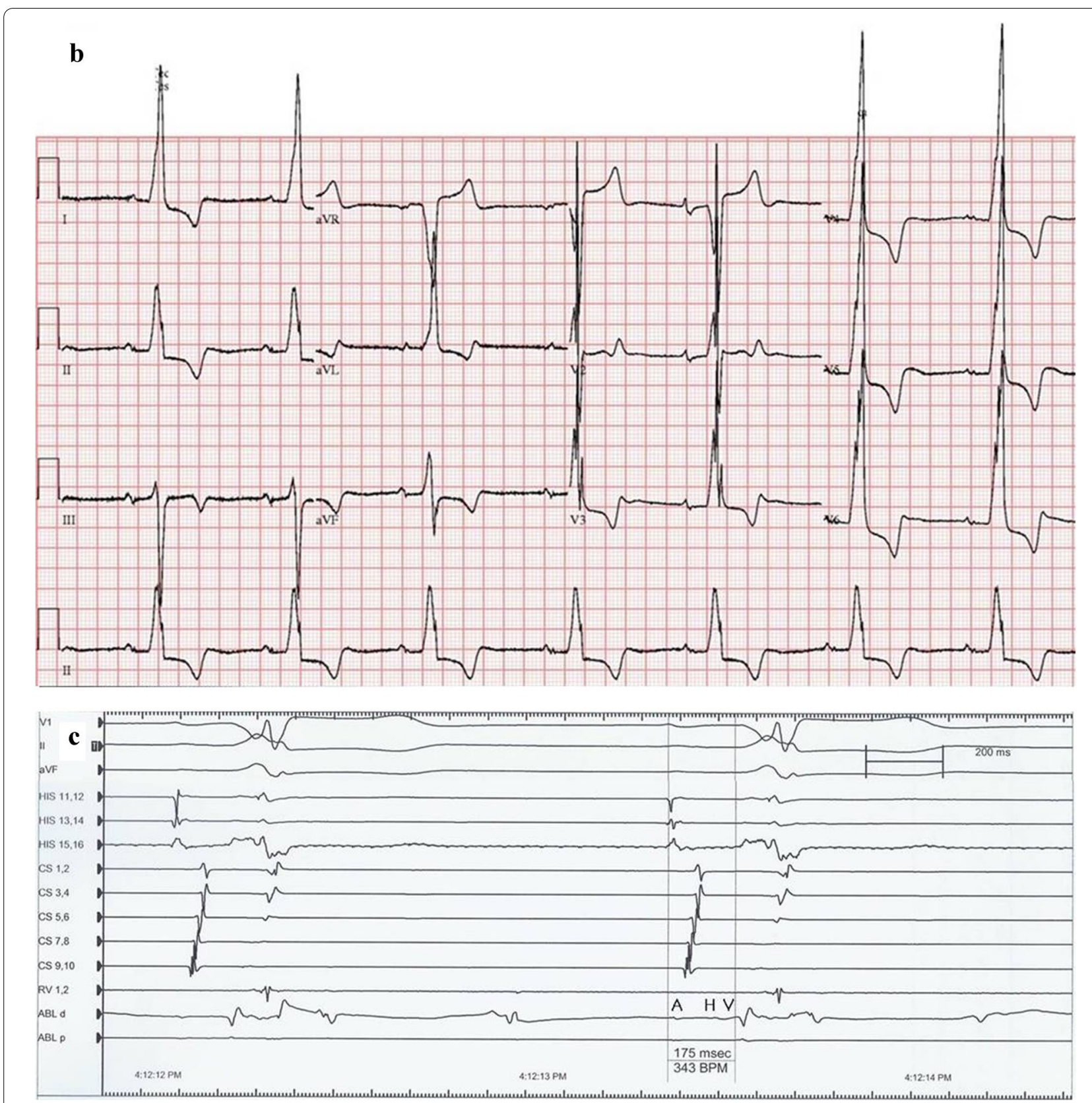

Fig. 3 continued

\section{Conclusion}

In this case, incidental injury on the AV conduction system revealed delta-like wide QRS origin from the HCM, but this method should be avoided except in selected patients such as planned a pacemaker implantation or AV node ablation. Further methods or clues for differentiation of delta-like wide QRS origin between HCM and WPW syndrome will be required.

\section{Abbreviations}

A: Atrium; ABL: Ablation catheter (d: distal, p: proximal); AH: Atrial-His; AF: Atrial fibrillation; AV: Atrioventricular; BT: Bypass tract; CL: Cycle length; CS: Coronary sinus (9, 10: proximal site, 1, 2: distal site); H and HIS: His bundle; HCM: Hypertrophic cardiomyopathy; HV: His-ventricular; LAO: Left anterior oblique; LV: Left ventricular; RAO: Right anterior oblique; Ref: Reference catheter; RV: Right ventricle; V: Ventricle; WPW: Wolff-Parkinson-White.

\section{Acknowledgements}

Not applicable. 


\section{Authors' contributions}

SHS and KHK were major contributors in writing the manuscript. JP designed the image, YJS and DKK contributed to the conception, and DIK revised the manuscript. All authors read and approved the final manuscript.

\section{Funding}

Not applicable.

\section{Availability of data and materials}

Single case report. Only clinical records were available.

\section{Declarations}

Ethics approval and consent to participate

Single case report. Not applicable.

\section{Consent for publication}

Written informed consent for publication of their clinical details and/or clinica images was obtained from the patient.

\section{Competing interests}

The authors declare no conflict of interests for this article.
Received: 15 January 2021 Accepted: 4 April 2021

Published online: 16 April 2021

\section{References}

1. Kelly BS, Mattu A, Brady WJ. Hypertrophic cardiomyopathy: electrocardiographic manifestations and other important considerations for the emergency physician. Am J Emerg Med. 2007:25:72-9.

2. Perosio AM, Suarez LD, Bunster AM, et al. Pre-excitation syndrome and hypertrophic cardiomyopathy. J Electrocardiol. 1983:16:29-40.

3. Song C, Guo Y, Zheng X, et al. Prognostic significance and risk of atrial fibrillation of Wolff-Parkinson-White syndrome in patients with hypertrophic cardiomyopathy. Am J Cardiol. 2018;122:1546-50.

4. Kottkamp $H$, Hindricks $G$, Shenasa $H$, et al. Variants of preexcitationspecialized atriofascicular pathways, nodofascicular pathways, and fasciculoventricular pathways: electrophysiologic findings and target sites for radiofrequency catheter ablation. J Cardiovasc Electrophysiol. 1996;7:916-30
Ready to submit your research? Choose BMC and benefit from:

- fast, convenient online submission

- thorough peer review by experienced researchers in your field

- rapid publication on acceptance

- support for research data, including large and complex data types

- gold Open Access which fosters wider collaboration and increased citations

- maximum visibility for your research: over 100M website views per year

At BMC, research is always in progress.

Learn more biomedcentral.com/submissions 